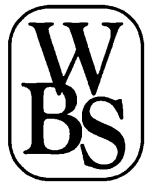

\title{
Fiberoptic airway management
}

\author{
Robin A. Stackhouse, MD \\ USCF, Department of Anesthesia and Perioperative Care, San Francisco General Hospital, \\ 1001 Potrero Avenue, San Francisco, CA 94110, USA
}

The use of fiberoptic scopes to assist in endotracheal intubation dates back to 1967, when a choledocoscope was used to intubate a patient with Still's disease [1]. The first case series of the use of fiberscopes for intubation was published in 1972 [2]. Their use in anesthesia greatly expanded after the publication of the ASA Guidelines on Difficult Airway Management [3]. Current uses include nasal and oral intubations (either in the awake or anesthetized patient), evaluation of the airway, verification of accurate placement of single-or double-lumen endotracheal tubes and laryngeal mask airways (LMA), endotracheal tube exchange, and placement of bronchial blocker devices.

Special skills are required to have a high degree of success with fiberoptic intubation. In teaching the technique, the author uses didactic sessions followed by a day-long workshop. Each participant has a fiberoptic bronchoscope (FOB), which allows unlimited time for practicing the mechanical and visual skills required to achieve a predictable degree of success with the technique. Many of the concepts and mechanical skills required for successful use of the FOB can be learned through the use of models [4,5] (Figs. 1,2). The "Hit the Hole" and "Tubes and Trachea" models were designed at the author's institution to emphasize concepts optimally and generate the necessary skills to use the FOB in clinical practice. The mechanical skills involved are as follows:

- Developing an appropriate grip on the FOB to achieve $360^{\circ}$ of rotation by the use of both directions of flexion.

- Rotating the FOB while remembering to keep the scope taut. If the scope is lax, the torque is not optimally translated to the tip, which results in a lesser change in the view through the eyepiece than would be attained if tension were maintained on the insertion cord.

- Moving the scope longitudinally. One should advance toward the object or back off and readjust the channel if the target is not achievable with the current orientation. 


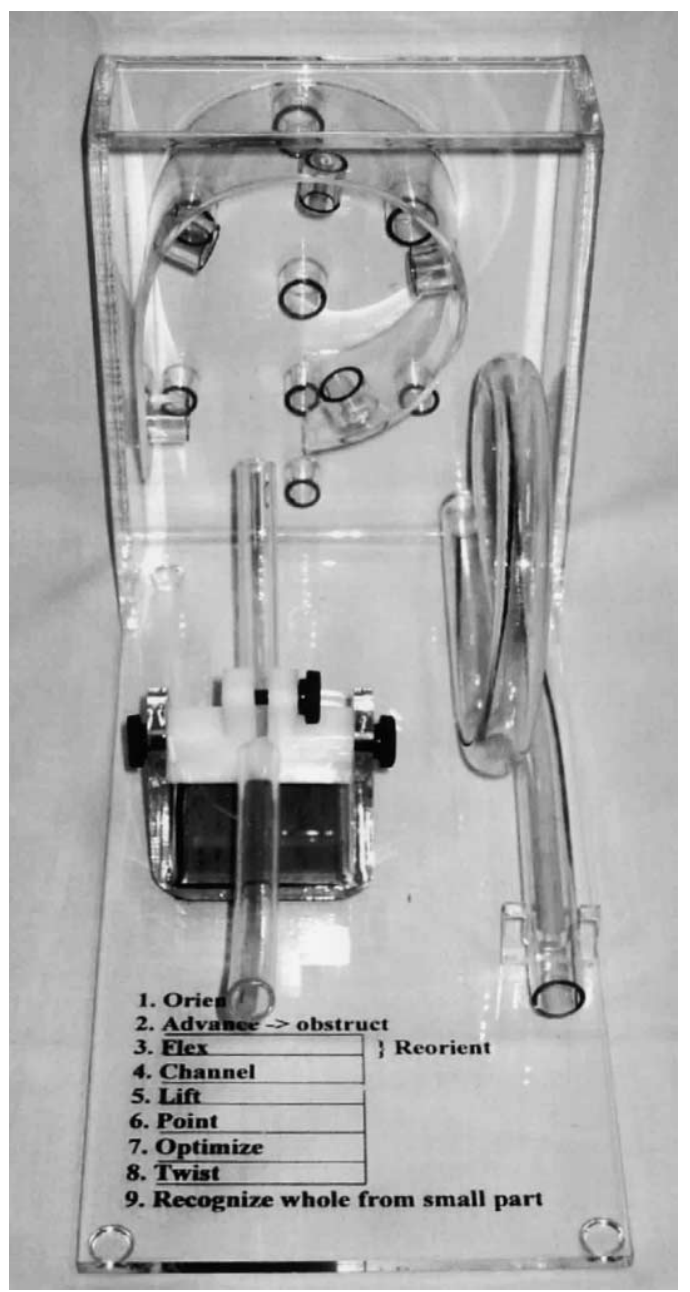

Fig. 1. Hit the hole model. This model is designed to improve the mechanical and visual skills needed to perform fiberoptic tracheal intubation successfully. It emphasizes the importance of an appropriately aimed channel. (From Bainton C. New concepts in airway management. Boston: Little, Brown and Co.; 1994; with permission.)

- Manipulating the channel. In clinical practice, the endotracheal tube (ETT) generally serves as the channel for nasal intubations. An intubating airway is used for oral intubations. The model allows for all the same manipulations of the channel that are possible or necessary for actual intubations. The channel can be rotated to adjust for aiming laterally. It can be advanced toward the object or pulled back to regain a broader field of view. It also can be aimed in a more anterior direction. In the clinical setting, this can be accomplished by inflating the cuff of the ETT to lift it off the posterior pharyngeal wall and aim it at the trachea, which has been described for 


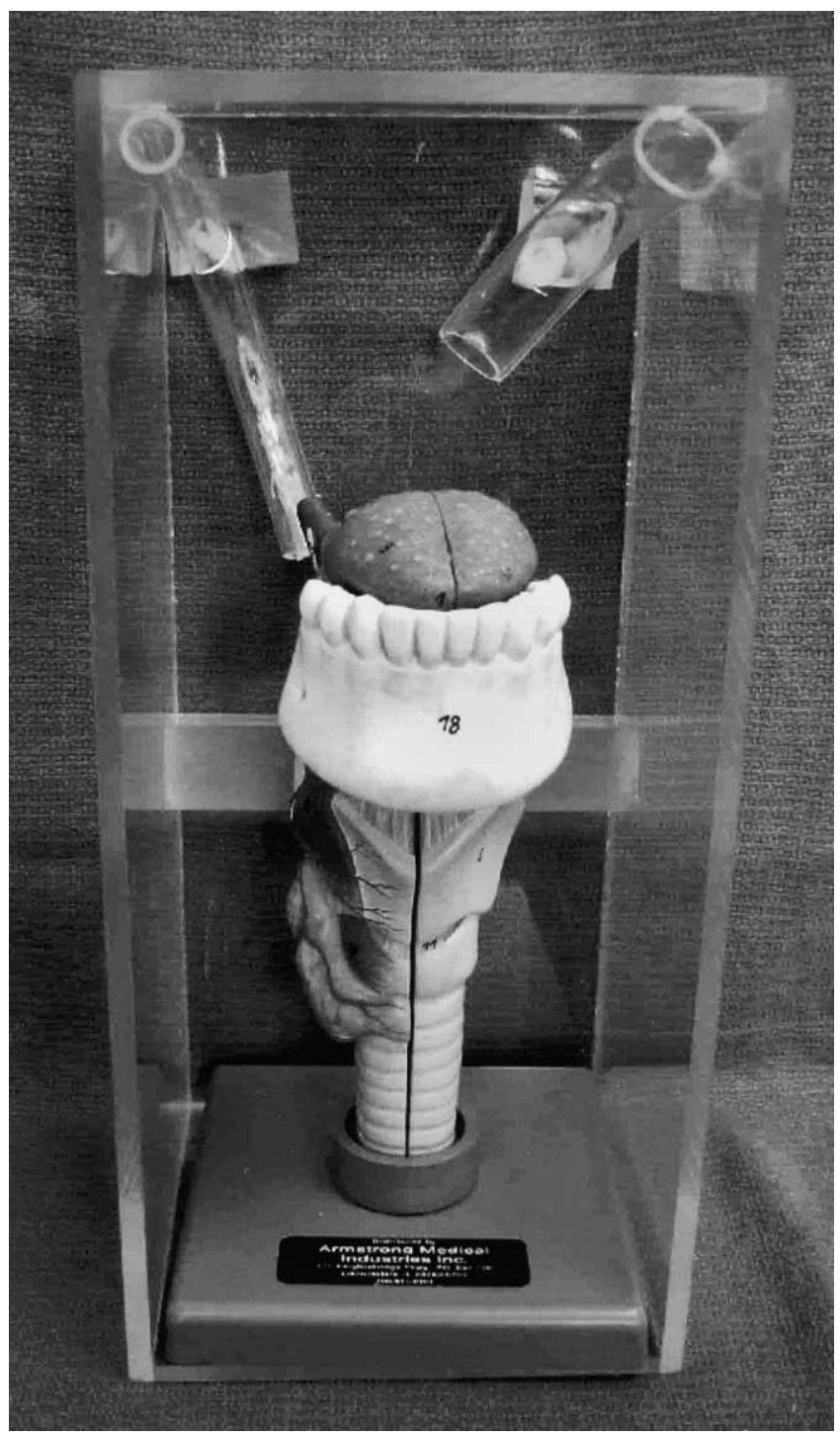

Fig. 2. Tubes and trachea model. The tube on the right is placed such that a fiber inserted into it does not reach the larynx. On the left, the tube is positioned to point directly at the larynx. Students practice by introducing the fiber through these channels. (From Bainton C. New concepts in airway management. Boston: Little, Brown and Co.; 1994; with permission.) 
facilitating blind nasal intubation [6-8]. Alternatively, anterior displacement of the tip of the ETT can be accomplished by traction on the pulley of an Endotrol tube (Mallinekrodt Company, St. Louis, MD).

The visual skills required are as follows:

- Realizing that to adjust the fiberoptic view, the FOB must be manipulated rather than one's own position, because the image is generated from the distal end of the optical fibers in the insertion cord of the FOB.

- Deciphering the fiberoptic image. The view is upright, upside-down, or sideways depending on whether one is at the head of the bed or in front of the patient and the orientation of a camera attached to the FOB (optional).

- Determining field of view through the FOB. It can be difficult to identify structures when too close to them and only a small portion is visible. It may be necessary to withdraw the FOB a certain distance to identify landmarks before advancing again.

- Reestablishing a view if the FOB becomes obscured by secretions or the tip fogs. Options include wiping the tip on the pharyngeal wall, injecting through the channel, or removing the scope from the airway and cleaning the tip.

- Learning to manipulate the channel to advance toward the airway based on the view seen through the FOB.

\section{Fiberoptic endoscopes}

\section{Physical characteristics}

The optical characteristics of fine glass strands are what make fiberoptic technology possible. When glass rods are heated and stretched to diameters of 8 to $25 \mu$, a flexible fiber capable of transmitting light is formed. By comparison, a human hair is approximately $20 \mu$. Light that enters the distal end of the fiber is reflected off the walls until it emerges at the proximal end. To keep a fraction of the light from being lost during transmission, the fiber is clad in a layer of glass that has a different optical density. This process results in total internal reflection of light entering the fiber. The light transmitted along a single fiber emerges uniformly distributed over the cross-sectional area at the end. The resolution of a fiberoptic image is inversely proportional to the diameter of the glass fiber [9]. To generate an actual image of an object, approximately 10,000 individual fibers are bound into a bundle, which can result in the microscopic equivalent of a cubist painting unless each individual fiber is in the exact same orientation at one end of the fiber as at the other (a coherent bundle). This is akin to the way one's retina or the individual pixels on a computer screen forms an image. The FOB contains another set of fiberoptic bundles that transmit light to the end of the insertion cord for illumination. These fiberoptic bundles do not need to be arranged in a coherent fashion. 


\section{Components of a fiberoptic endoscope}

The FOB is composed of an eyepiece, a control section, the insertion cord, and a universal cord (or a miniature light source) (Fig. 3).

\section{Eyepiece and control section}

The eyepiece contains the lenses and is focused using the diopter adjustment ring. The instrument has a depth of field from a few millimeters $[3,4]$ to approximately $50 \mathrm{~mm}$. The scope should be cleaned, focused, and defogged before use. Once the scope is focused for the user's visual acuity, it does not need additional focusing. If the view becomes obscured, it is because it has become soiled with secretions or blood or was inadequately defogged.

The control section contains the angulation control lever and, for scopes with a suction or biopsy channel, the connectors and ports are in this section. The control lever causes the distal tip of the insertion tube to flex in the plane indicated by a marker seen through the eyepiece. By combining flexion and rotation, it is possible to traverse and visualize $360^{\circ}$ despite the $90^{\circ}$ limitation of the scope's field of view. The scope never should be flexed forcibly because it can damage the control mechanism. The suction port can be used for instilling solutions (generally local anesthesia) directly on the visualized structures. Some clinicians use the port to insufflate supplemental oxygen during endoscopy. Some of the oxygen is swallowed, however, which may lead to gastric distention. There is one case report of gastric rupture that was believed to have been caused by oxygen insufflation through the channel [10]. The port is designed to attach to a suction device to remove secretions. This feature can be problematic because the channels of the scopes generally used for fiberoptic intubation ( $\leq 4 \mathrm{~mm}$ outer diameter [OD]) have small diameters, which limits their effectiveness when the secretions are viscous and possibly soiling the tip of the optical bundles.

There also may be an ethylene oxide vent on this portion of the FOB. Alternatively, it may be located on the universal cord. Venting is required during gas sterilization to equalize internal and external pressures.

\section{Insertion cord}

The outer sheath of the insertion cord encases the optical and the illuminating fiberoptic bundles, the angulation wires, and the channel. The fiberoptic bundles are fragile, which requires that the insertion cord be treated with care. Any breakage of the illuminating fiberoptic bundles decreases the amount of light that reaches the tip of the scope. Breakage of optical fibers results in black spots in the image because those pixels of data are lost.

\section{Universal cord}

The universal cord contains the fiberoptic bundles, which transmit light from the light source to the insertion cord. There are currently fiberoptic scopes that incorporate a miniature battery-operated light source that attaches to the control 


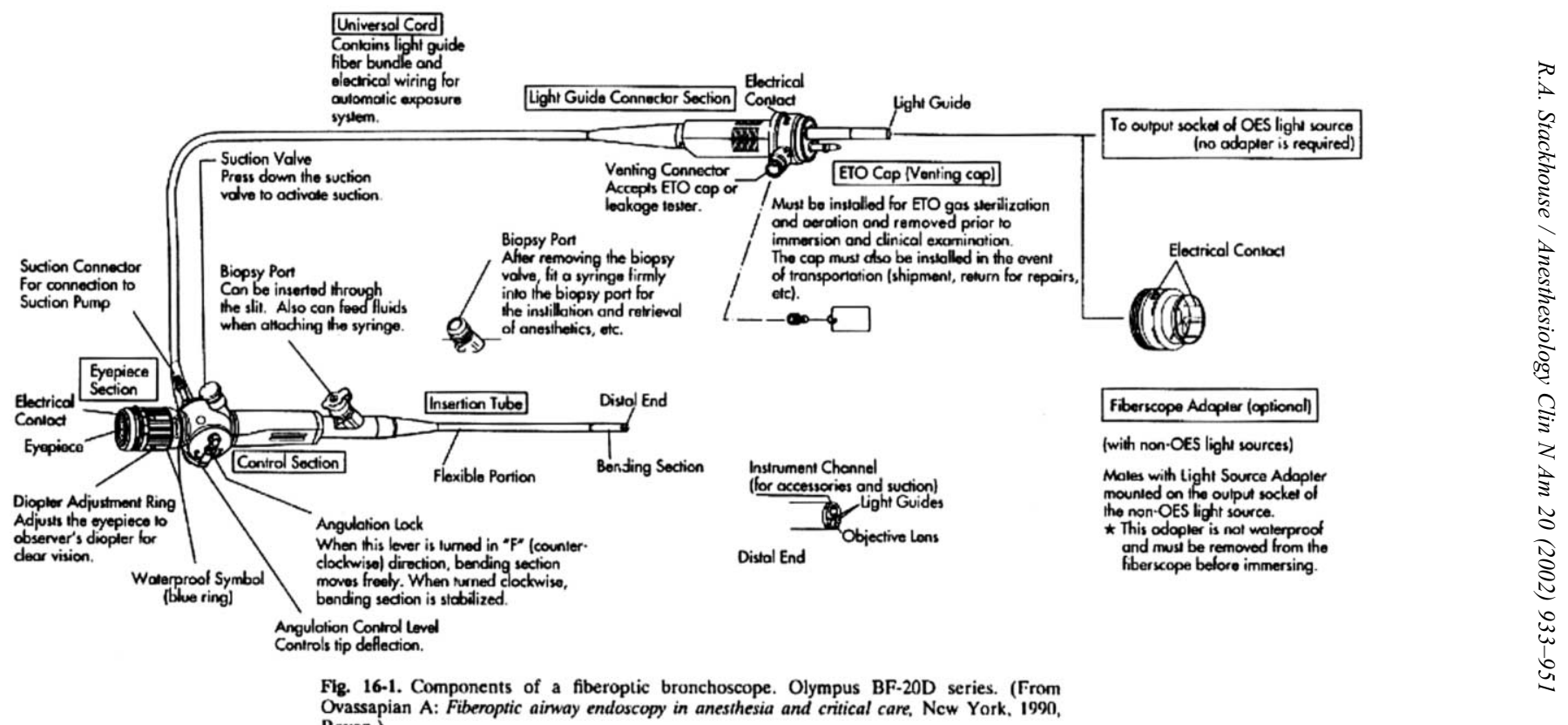

Fig. 3. Fiberoptic bronchoscope. Components of a fiberoptic bronchoscope. (From Ovassapian A. Fiberoptic airway endoscopy in anesthesia and critical care. New York: Raven Press; 1990; with permission.) 
section of the FOB. This feature makes the scope far more transportable for use outside the operating room.

\section{Fiberoptic intubation}

\section{Indications}

Fiberoptic endotracheal intubation is most frequently chosen when it is anticipated or known that a patient is difficult to intubate on direct laryngoscopy. It is ideally suited to these situations because the technique can be performed before inducing general anesthesia. The risks of failed intubation and ventilation can be all but eliminated. This elimination is predicated on the assumptions that there is adequate time to perform the technique and that the patient is never sedated inappropriately. The causes of difficult endotracheal intubation fall into four main categories: limited oropharyngeal space, decreased atlanto-occipital extension, decreased pharyngeal space, and decreased submandibular compliance $[4,11-13]$.

\section{Oropharyngeal space}

Oropharyngeal space may be limited in many chronic and acute conditions. Decreased space, as with a naturally small mouth, temporomandibular joint disease, or a mouth that is contracted from scarring (burns, surgery, betelnut use) or is relatively small because of protuberant teeth or abnormal tissue (masses, edema, infection) may limit space for visualization on direct laryngoscopy. This contributes to an inability to optimally align the oral and pharyngeal axes on direct laryngoscopy. These conditions can be identified when performing the Mallampati examination $[14,15]$.

\section{Atlanto-occipital extension}

Extension of the head on the neck at the atlanto-occipital joint begins to bring the oral and pharyngeal axes into alignment as required for visualization of the airway structures during direct laryngoscopy. Limitation of atlanto-occipital extension of more than one third, from a norm of $35^{\circ}$, is associated with difficult laryngoscopy [16].

\section{Pharyngeal space}

Pharyngeal space for aligning and visualizing laryngeal structures is limited when soft tissue fills the area. This condition occurs in patients who have a relatively small mandible (decreased thyromental distance) in relation to the size of the tongue and in patients with abnormalities of the soft tissues (infections, edema, hematoma, tumors). Although external abnormalities that limit pharyngeal space are easily assessed, internal anomalies may be subtle to diagnose. One must rely on clinical signs such as assessory muscle use, patient problems with managing secretions, the preferred position of the patient (upright with head and 
neck in the "sniffing" position, as with epiglottitis), or the restriction of maximal inspiratory airflow.

\section{Submandibular compliance}

The ability to displace the soft tissues of the pharynx anterior to the line of vision on direct laryngoscopy depends on the compliance of the submandibular tissue. Conditions such as infection (Ludwig's angina), tumor infiltration, and scarring can decrease the compliance of the tissue so severely that the airway structures cannot be visualized.

\section{Indications}

Fiberoptic intubation is ideally suited for use in patients with unstable cervical spines. The technique does not require any movement of the neck and can be performed before induction of general anesthesia, which allows for evaluation of the patient's neurologic function after intubating and surgical positioning.

Patients who have had an injury to the airway-either from blunt or penetrating trauma - are at risk of the endotracheal tube creating a false passage by exiting the airway through the disrupted tissue when intubated with direct laryngoscopy. By performing a fiberoptic intubation, not only can the injury be assessed but also the tube can be placed beyond the level of the injury. This approach also eliminates the risk of causing subcutaneous emphysema that could compress and further compromise the airway when positive pressure ventilation forces air into the tissues through the injury.

\section{Contraindications}

An absolute contraindication to fiberoptic intubation is lack of time. The technique requires time to set up the equipment and prepare the patient's airway for the intubation. If immediate airway management is required, another technique should be used. Several circumstances make fiberoptic intubation relatively contraindicated, because the chance of success is diminished or it poses certain risks for the patient. Because the field of view through a FOB depends on the presence of space around the scope, anything that impinges on the lumen makes the intubation more difficult. Some common causes include edema of the pharynx or tongue, tracking infections, hematoma, and infiltrating masses. Unlike in laparoscopic procedures, in which $\mathrm{CO}_{2}$ can be insufflated to maintain space around the scope, the pharynx cannot be distended in this way because it is in direct communication with atmospheric pressure. There are other ways of generating space, however. The usual means of airway support can be used, as can inflating the cuff of the ETT to hold open the pharyngeal walls. Blood and secretions easily soil the optics of the FOB. An inability to keep the tip clean results in failure. Giving an antisialagogue, suctioning, and maintaining pharyngeal space can minimize soiling. Another relative contraindication to fiberoptic intubation is the presence of a pharyngeal abscess, which can be disrupted as the ETT is advanced over the FOB and can result in the aspiration of purulent material $[9,13,17,18]$. 


\section{Clinical aspects}

Once it is determined that fiberoptic intubation is the appropriate choice, one must decide whether to use a nasal or oral approach and whether to keep the patient awake until control of the airway is established or to perform the technique with the patient anesthetized. In general, the nasal route is easier for fiberoptic intubation because the angle of curvature of the ETT (channel) naturally approximates that of the patient's upper airway, hence the reason it is possible to perform blind nasal intubations. When performing an oral fiberoptic intubation, an alternative channel that creates more anterior curvature is required, which is accomplished by using one of the commercially available intubating oral airways (Fig. 4). The gag reflex is less pronounced with nasal intubation than with oral, but adequate topical anesthesia and local anesthetic blocks can overcome it. The risk of causing bleeding is higher when the nasal route is used, however, and it is relatively contraindicated in patients with coagulation disorders. Oral fiberoptic intubation is also indicated in patients who have a contraindication for nasal application of vasoconstrictors (ie, parturients, some heart disease patients). The decision to perform the procedure in an awake versus anesthetized patient depends on the risk of losing airway control. It is safest to have the patient maintain his or her own oxygenation and ventilation if there is

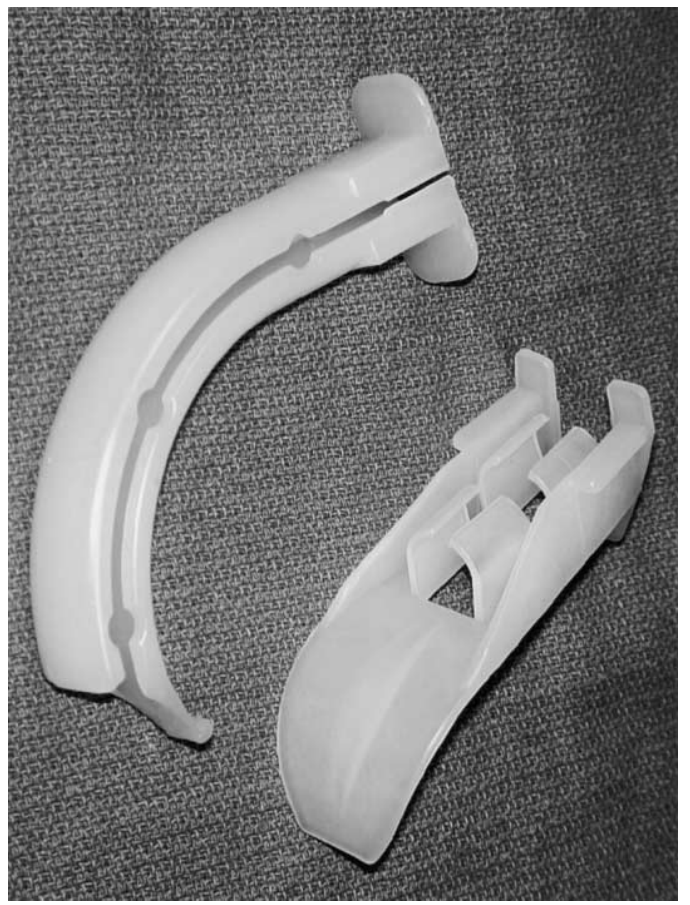

Fig. 4. Berman and Ovassapian oral intubating airways. 
any concern about one's ability to manage the patient's airway. One should not give up wakefulness easily.

\section{Patient preparation}

The following description concentrates predominantly on the technique for awake nasal fiberoptic intubation. The differences in technique for awake oral, asleep nasal, and asleep oral are discussed. The procedure should be explained to the patient along with assurance that he or she will be made as comfortable as possible. An antisialagogue (0.2 $\mathrm{mg}$ IV glycopyrrolate) should be administered preoperatively to inhibit the formation of secretions that would make it more difficult to perform the fiberoptic intubation. Sedation choices are numerous. The author generally uses a combination of midazalam (anxyolysis, amnesia), and fentanyl (analgesia). Topical anesthesia and local anesthetic blocks are then administered.

\section{Topical anesthesia and nerve blocks of the upper airway}

Nose and nasopharynx. The ophthalmic (V1) and maxillary (V2) divisions of the trigeminal nerve (CNV) provide innervation to the nasal mucosa as the anterior ethmoidal nerve, the nasopalatine nerve, and the sphenopalatine ganglion [12,13,17-19] (Fig. 5). The nasal mucosa must be anesthetized and vasoconstricted, which is typically performed with either a $4 \%$ cocaine solution or a combination of $3 \%$ lidocaine $/ 0.25 \%$ phenylephrine. Conflicting data exist regarding absorption and physiologic consequences of these agents. This could be because of the manner of application, the dose, and the duration of mucosal contact. There are certain patient populations in which these agents should be used with care, including parturients, who are at risk of decreased uteroplacental perfusion with either of the solutions, and certain patients with cardiovascular disease, who may develop myocardial ischemia from coronary artery vasospasm with cocaine or who would be unable to tolerate increased systemic vascular resistance, which may occur with either agent [20-29]. The solutions can be applied on soaked cotton swabs or pledgets. One nostril may have a larger passage than the other, and that side should be used to decrease the likelihood of bleeding when the ETT is passed through the nose. To avoid bleeding and discomfort, force should never be used if resistance is encountered when applying the topical anesthesia or advancing the ETT. Bleeding can occur if an incorrect angle is used or the ETT is too large.

Tongue and oropharynx. The lingual nerve, which is a branch of the mandibular division (V3) of the trigeminal nerve, innervates the anterior two thirds of the tongue. The posterior one third is innervated by the glossopharyngeal nerve (CN IX), which also provides branches to the soft palate and the oropharnyx (Figs. 6,7). Topicalization may be achieved by aerosolized local anesthesia or by bilateral block of the glossopharyngeal nerve at the base of 


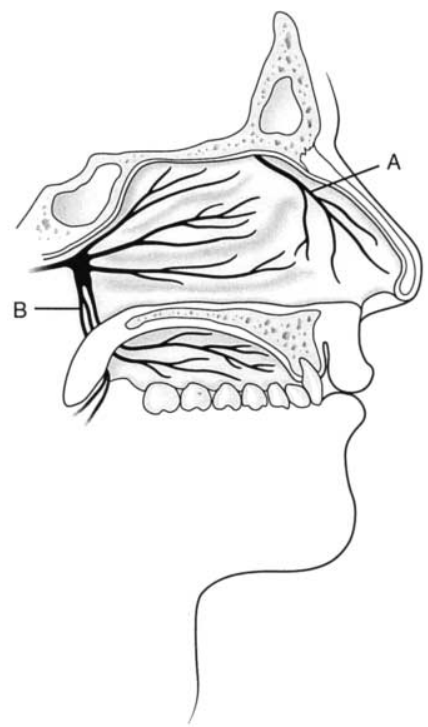

Fig. 5. Innervation of the nasal cavity. Diagram of the lateral wall of the nasal cavity illustrates its sensory nerve supply. Anterior ethmoidal nerve, a branch of the ophthalmic division of the trigeminal nerve, supplies the anterior third of the septum and the lateral wall (A). The maxillary division of the trigeminal nerve via the sphenopalatine ganglion supplies the posterior two thirds of the septum and the lateral wall (B). (From Ovassapian A. Fiberoptic airway endoscopy in anesthesia and critical care. New York: Raven Press; 1990; with permission.)

each anterior tonsillar pillar. Approximately $2 \mathrm{~mL}$ of $2 \%$ lidocaine injected at a depth of $0.5 \mathrm{~cm}$ is sufficient. It is necessary to aspirate on the syringe before injecting to ensure that the needle has not gone through the pillar, which results in air return, or intravascularly. With the spray technique, peak blood concentrations occur at 30 to 40 minutes after mucosal application. Despite potentially high total doses, lidocaine is unlikely to cause local anesthetic toxicity except in patients with liver disease.

Larynx and trachea. The vagus nerve (CNX) branches into the superior laryngeal nerve and the recurrent laryngeal nerve, both of which supply innervation to the airway. The internal branch of the superior laryngeal nerve provides sensation to the lower pharynx and the larynx above the true vocal cords, whereas the recurrent laryngeal nerve provides sensation to the vocal cords and trachea. The recurrent laryngeal nerve supplies the motor innervation to all the intrinsic muscles of the larynx except the cricothyroid muscle, which is innervated by the external branch of the superior laryngeal nerve (Fig. 8).

Topicalization or nerve blocks may be used for the larynx and trachea. Local anesthesia may be sprayed, aerosolized, or nebulized into the airway. One should note that the larger particle size of a spray tends to rain out in the pharynx, with only a small proportion reaching the trachea. Conversely, the small particle size of a nebulized spray is carried more effectively into the trachea but also the 


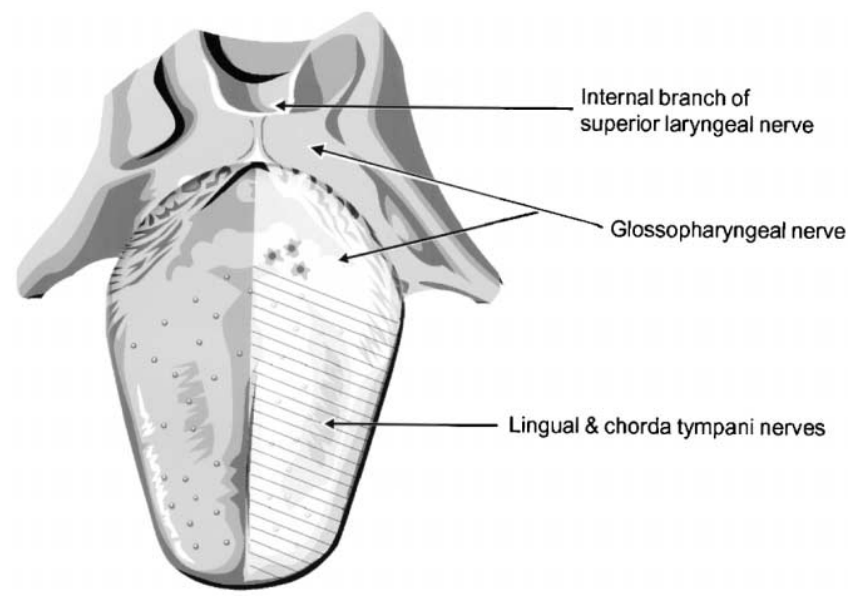

Fig. 6. Sensory innervation of the tongue.

smaller airways, where the anesthesia is not needed and has a more rapid systemic absorption. Lidocaine has a wide therapeutic window, and toxicity is rare when it is used for airway topicalization. Benzocaine has been shown to cause methemoglobinemia even in therapeutic doses. Tetracaine has a narrow therapeutic window. The maximum allowable dose can easily be exceeded. Cetacaine is a mixture of benzocaine and tetracaine.

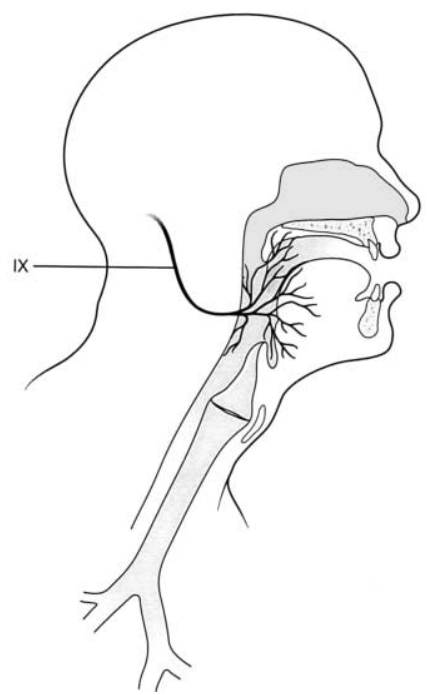

Fig. 7. Glossopharyngeal nerve. Sensory distribution of the glassopharyngeal nerve (CN IX). (From Patil VU, Stehling LC, Zander HL. Fiberoptic endoscopy in anesthesia. St Louis: CV Mosby; 1983; with permission.) 


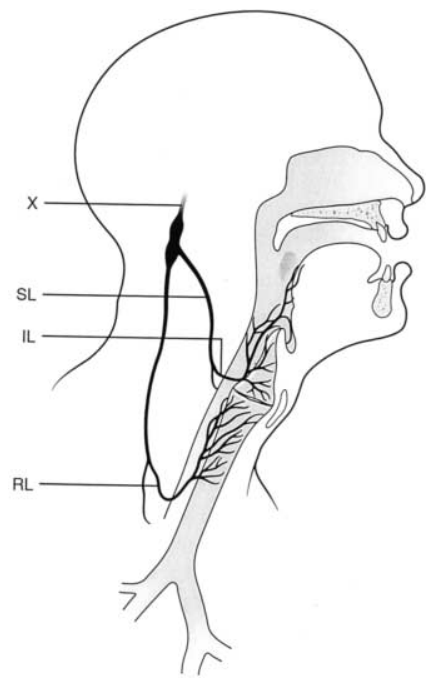

Fig. 8. Vagus nerve. Sensory distribution of the vagus nerve (CN X). (From Patil VU, Stehling LC, Zander HL. Fiberoptic endoscopy in anesthesia. St Louis: CV Mosby; 1983; with permission.)

The internal branch of the superior laryngeal nerve is blocked by instilling local anesthesia, bilaterally, in the vicinity of the nerves where they lie between the greater cornu of the hyoid bone and the superior cornu of the thyroid cartilage as they traverse the thyrohyoid membrane to the submucosa of the pyriform sinus (Fig. 9). The overlying skin is cleaned with alcohol or betadine, and either the cornua of the hyoid or the thyroid may be used as a landmark. A 22- to 25-gauge needle is "walked" off the cephalad edge of the thyroid or the caudal edge of the

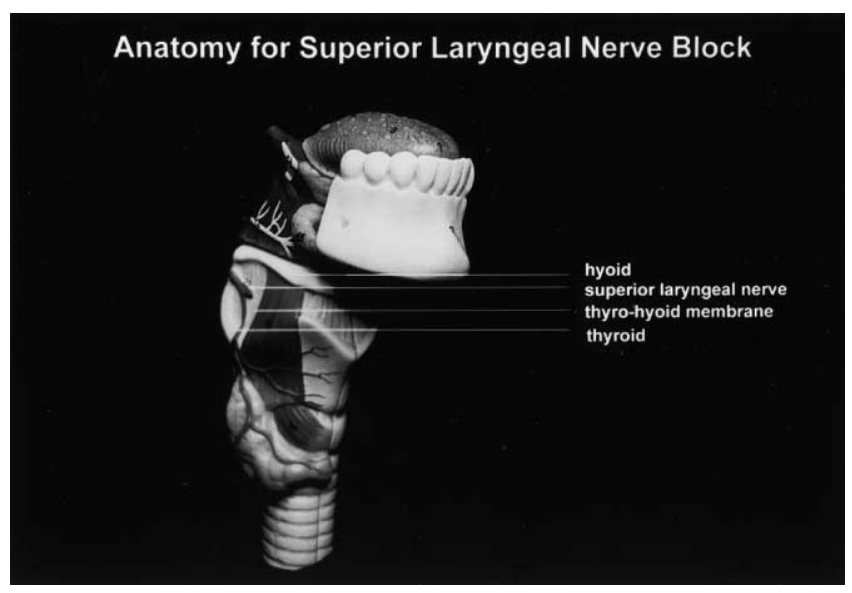

Fig. 9. Anatomy for superior laryngeal nerve block. 
hyoid, and approximately $2 \mathrm{~mL}$ of local anesthesia is injected. For transtracheal block, a prominent thyroid cartilage is the landmark of choice. If it is not easily identifiable, the cricoid cartilage can be recognized as a wider and higher cartilage than those below it. The skinned is prepped with alcohol or betadine and a 20-gauge intravenous catheter is advanced through the cricothyroid membrane while simultaneously aspirating on a syringe, filled with $4 \mathrm{~mL}$ of local anesthesia, that is attached to its end. When air is aspirated, the needle is withdrawn, the syringe is reattached to the catheter, aspiration of air is reconfirmed, and the local anesthesia is injected. The author prefers an intravenous catheter to a needle because there is less risk of causing injury to the trachea if the patient coughs during injection.

\section{Fiberoptic laryngoscopy}

Once the airway is anesthetized, a lubricated ETT that is at least $1.5 \mathrm{~mm}$ larger than the diameter of the FOB is advanced through the nose into the pharynx. Softening the ETT in warm water before use makes it less likely to cause mucosal trauma or submucosal tunneling. The ETT must be aimed appropriately (perpendicular to the plane of the face). $90^{\circ}$ counter-clockwise rotation of the ETT can help it take the turn at the posterior nasopharynx if resistance is encountered. Pharyngeal secretions should be suctioned before inserting the FOB through the ETT. One should follow the stripe on the ETT to the beveled end to ensure that the FOB does not exit through the Murphy eye. Ideally, the vocal cords are visualized just beyond the tip of the ETT. Sometimes, instead of the vocal cords or some other recognizable airway structure, only secretions or soft tissue is seen. This occurrence requires manipulation of the channel. Frequently, the solution is to create space in the pharynx, which is accomplished easily by inflating the ETT cuff. This approach also aims the ETT anteriorly.

Once a field of view is established, it can be determined whether the channel is correctly positioned to aim the FOB toward the airway or whether it must be advanced or withdrawn. The target always should be kept in the center of the view by flexion and rotation as the FOB slowly advances. As the FOB passes through the vocal cords, the tracheal rings become visible. The scope is advanced to just above the carina and then the ETT is threaded over the scope. If resistance is felt when advancing the ETT, force should not be exerted. Force can kink the FOB and result in an ETT in the esophagus and a damaged FOB. (The ETT is probably caught on a vocal cord and can be released by rotating the tube as it is gently advanced.) The appropriate depth of the tube can be verified by observing the distance between the carina and the tip of the ETT as the scope is withdrawn. If there is any resistance when removing the FOB, it is either out the Murphy eye or kinked in the pharynx. In both instances, the ETT and the scope must be withdrawn together to avoid damaging the scope.

When performing an awake oral fiberoptic intubation, the airway is anesthetized, omitting the nasal topicalization. The curvature of the ETT is not optimal for oral intubation. The use of an oral intubating airway serves as a more effective channel. The airway can work against you if it is either too large or small. One 
must exercise care to maintain it in a midline position. Alternatively, a laryngeal mask airway provides an excellent channel for oral fiberoptic intubation.

Fiberoptic intubation under general anesthesia should be considered only if adequate oxygenation and ventilation can be maintained. Nasal and oral intubation is possible, and the technique can be performed with the patient breathing spontaneously or while ventilation is controlled. A nasal airway can be placed and connected to the anesthesia circuit via a $15-\mathrm{mm}$ connector. When providing an airway in this manner, it is preferable to use an intravenous anesthetic technique, because persons in the room (particularly the laryngoscopist) are exposed to the anesthetic vapors if volatile agents are insufflated to maintain anesthesia.

Another option is to use a Patil-Syracuse mask (Anesthesia Associates, Inc., San Marcos, CA) (Fig. 10). The mask is designed with a port that accommodates an ETT and a FOB through a diaphragm. It allows for spontaneous or controlled ventilation while fiberoptic nasal or oral intubation is performed. The predominant difference in performing the technique in an anesthetized patient is that the soft tissues of the pharynx tend to relax and limit space for visualization with the FOB. Some mechanism must be used to generate the requisite space to allow for a field of view. One can accomplish this task by using jaw thrust or a tonsil retractor, expanding the ETT cuff in the pharynx, or applying traction on the tongue. It is advisable to have a second person trained in anesthesia delivery assisting when a fiberoptic intubation is performed under general anesthesia, because it is difficult to maintain a patient's airway, be attentive to the monitors, and perform the fiberoptic intubation alone. When using the nasal approach, topical anesthesia for the nasal mucosa is not requisite, but vasoconstriction to increase the diameter of the passage and decrease the risk of bleeding is

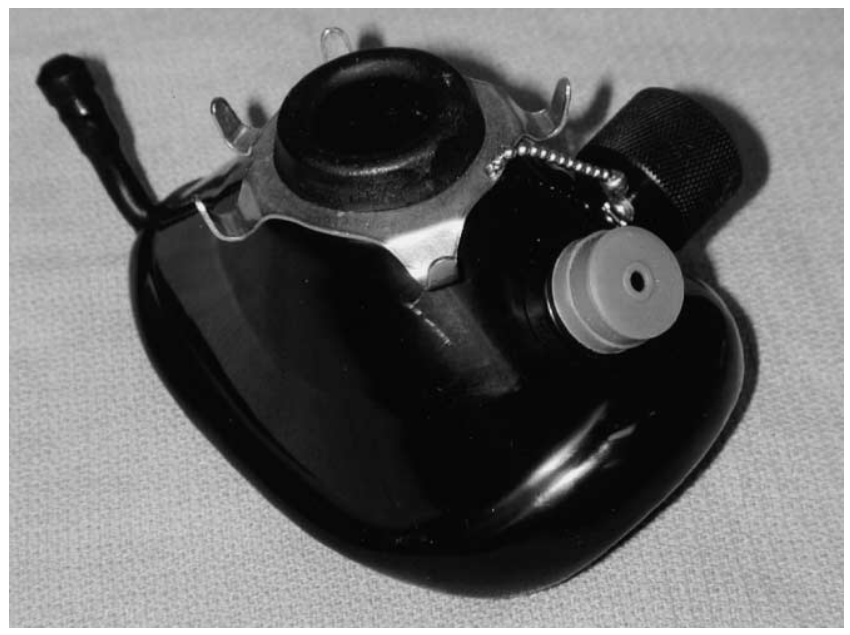

Fig. 10. Patil-Syracuse mask. 
necessary. For the nasal or the oral approach, topical anesthesia or blocks to inhibit the reflexes of the pharynx, vocal cords, and trachea are useful. The plane of anesthesia tends to be light, and if these reflexes are still intact, the patient may cough, move, develop laryngospasm, or reflux gastric contents.

\section{Fiberoptic essentials}

Fiberoptic intubation is a complex technique. Failure may occur for several reasons, which may be condensed into 11 essentials [9]:

\section{Elements that require forethought}

1. Choose the right patient. This choice requires an understanding of the indications and contraindications to the technique.

2. Allow sufficient time. The technique takes time even when expertly executed.

3. Allow sufficient pharyngeal space. A field of view for manipulating the FOB into the airway is necessary.

4. Ensure minimal pharyngeal blood and secretions. An antisialagogue can decrease further secretion production, and appropriate use of a vasoconstrictor agent and gentle technique can decrease iatrogenic bleeding. Patients with uncontrolled bleeding into the upper airway are poor candidates for this technique.

\section{Elements that relate to the fiberoptic bronchoscope}

5. Clean, focus, and defog the FOB before use.

6. Use a channel. The insertion cord of the FOB is malleable and tends to accordion when it encounters resistance. A channel serves to provide a supporting exoskeleton.

7. Aim the channel. Fiberoptic laryngoscopy is a two-component system: a FOB and a channel. Despite skillful manipulation of the scope and the ability to visualize the appropriate structures, it may be impossible to advance the scope to the targeted area. This inability occurs because the scope is supple and it advances along the long axis of the insertion cord. The appropriate channel aims the FOB toward the airway, minimizes the effort involved, and maximizes the chances of success.

\section{Elements that relate to the patient}

8. Maintain supplemental oxygen as required by a patient's medical condition and the level of sedation. The procedure must be abandoned if adequate oxygenation cannot be maintained.

9. Titrate sedative agents to make the procedure comfortable for the patient with the caveat that the level of sedation be inversely proportional to the tenuousness of the airway. 
10. Provide adequate anesthesia (topical anesthesia and nerve blocks) along the track that the FOB and ETT follow to eliminate pain, gag reflex, and coughing.

\section{Elements that relate to the laryngoscopist}

11. Ensure expertise. Skill with the FOB correlates with success. Skill can overcome many suboptimal clinical situations in which a less practiced clinician would fail. Practice with the technique on models and whenever clinically appropriate aids in acquiring the necessary proficiency.

\section{Care of the fiberoptic endoscope}

Fiberoptic endoscopes generally cost approximately $\$ 5000$. A single repair bill can cost more than $\$ 1500$. Care must be taken when using and processing these instruments or maintenance and repair can become costly and result in limited availability of the scopes for clinical use. When using the scope, it must be placed such that it is not at risk of being crushed in any way. Damage most frequently occurs when one of the cords hangs over the edge of a cart and a drawer crushes it as it is closed.

Processing the endoscope after each use is critical to avoid disease transmission to subsequent patients. All classes of microorganisms, including bacteria, fungi, and viruses (most recently hepatitis $\mathrm{C}$ virus), have been implicated [30-32]. The unifying feature in all the case reports of cross-contamination is that there was a lack of adherence to recommended practices of endoscope disinfection.

Fiberoptic endoscopes used for intubation come in contact with mucosal surfaces and are considered semi-critical devices by the E.H. Spaulding Classification of Patient Care Items, which require a minimum of high-level disinfection between uses. As soon as possible after using the FOB, all surfaces should undergo cleaning with a low-sudsing enzymatic detergent followed by rinsing to remove organic material. Organic material that is sequestered in the channel of the FOB has been associated most commonly with disease transmission. The channel should be irrigated with the detergent solution and a specially designed brush used to remove particulate matter. Disposable brushes should be used or they also should undergo high-level disinfection.

The FOB should be tested for leaks. If this step is omitted, it may convert a relatively inexpensive repair into a problem that requires replacement of the scope. A scope that does not pass the leakage test should not undergo the next steps in the cleaning process but should be sent for repair. After successful leak testing, the scope is either liquid or gas sterilized. The disinfecting agent must be in contact with all surfaces of the FOB for the recommended period of time. The Environmental Protection Agency-recommended agents for high-level disinfection include $2 \%$ alkaline or acid glutaraldehyde, $6 \%$ hydrogen peroxide $/ 0.85 \%$ phosphoric acid, and $1 \%$ peracetic acid. The FOB is most 
commonly processed by a Steris System (Steris Corporation, Mentor, OH) (peracetic acid) or ethylene oxide gas sterilization. After liquid processing, the external surfaces and channel of the scope should be rinsed with water. The channel then should have air aspirated through it for 60 seconds if sterile water was used for rinsing. If nonsterile water was used, the channel should have $70 \%$ alcohol suctioned through it. The scope is then allowed to dry. Each manufacturer of FOBs has specific recommendations for the processing of their scopes. The recommendations even may be specific for different scopes made by the same manufacturer. The recommendations should be followed so that the scope does not sustain damage or the warranty became voided [33-35].

\section{Summary}

Despite many recent innovations in equipment for difficult airway management, it remains the leading cause of the most devastating adverse outcomes in anesthesia. Fiberoptic airway management is among the most versatile of techniques for difficult airway management. It requires a greater degree of skill than most other techniques, however. Training, practice, and adherence to a few basic principles are required. It is most frequently used when difficult airway management is predicted but also may be appropriate when unforeseen difficulty arises. It cannot be too strongly emphasized, however, that this technique takes time and should be entertained only if the anesthesia care provider is able to maintain adequate oxygenation and ventilation until the airway is secured.

\section{References}

[1] Murphy P. A fibre-optic endoscope used for nasal intubation. Anaesthesia 1967;22:489.

[2] Stiles CM, Stiles QR, Denson JS. A flexible fiberoptic laryngoscope. JAMA 1972;221:1246.

[3] American Society of Anesthesiologists Task Force on the Difficult Airway. Practice guidelines for management of the difficult airway. Anesthesiology 1993;78:597-602.

[4] Bainton CR. Models to facilitate the learning of fiberoptic technique. Int Anesthesiol Clin 1994;32:47-55.

[5] Williams KA, Harwood RJ, Woodall NM, et al. Training in fibreoptic intubation. Anaesthesia 2000;55:99-100.

[6] Baraka A. Tracheal tube cuff inflation as an aid to blind nasotracheal intubation in a patient with cervical spine injury. Br J Anaesth 1993;71:772-3.

[7] Gorback MS. Inflation of the endotracheal tube cuff as an aid to blind nasal intubation [letter]. Anesth Analg 1987;66:917.

[8] Van Elstraete AC, Mamie JC, Mehdaoui H. Nasotracheal intubation in patients with immobilized cervical spine: a comparison of tracheal tube cuff inflation and fiberoptic bronchoscopy. Anesth Analg 1998;87:400-2.

[9] Marks JD, Bainton CR. Practical aspects of fiberoptic laryngobronchoscopy. Int Anesthesiol Clin 1994;32:31-46.

[10] Hershey MD, Hannenberg AA. Gastric distention and rupture from oxygen insufflation during fiberoptic intubation. Anesthesiology 1996;85:1479-80.

[11] Bainton CR. Difficult intubation: what's the best test? Can J Anaesth 1996;43:541-3. 
[12] Stackhouse R, Bainton CR. Difficult airway management. In: Hughes SC, Rosen M, Levinson G, editors. Anesthesia for obstetrics. $4^{\text {th }}$ edition. Baltimore: Williams and Wilkins; 2001. p. 375-90.

[13] Stackhouse R, Marks JD, Bainton CR. Performing fiberoptic endotracheal intubation: clinical aspects. Int Anesthesiol Clin 1994;32:57-73.

[14] Mallampati SR, Gatt SP, Gugino LD, et al. A clinical sign to predict difficult intubation: a prospective study. Can Anaesth Soc J 1985;32:429-34.

[15] Mallampati SR. Clinical sign to predict difficult tracheal intubation (hypothesis). Can J Anaesth 1983;30:316.

[16] Bellhouse CP, Dore C. Criteria for estimating the likelihood of difficulty of endotracheal intubation with the Macintosh blade. Anaesth Intensive Care 1988;16:329-37.

[17] Ovassapian A, Wheeler M. Fiberoptic endoscopy-aided techniques. In: Benumof J, editor. Airway management: principles and practice. St. Louis: Mosby-Year Book; 1996. p. 282-319.

[18] Ovassapian A. Fiberoptic tracheal intubation. In: Fiberoptic airway endoscopy in anesthesia and critical care. New York: Raven Press; 1990. p. 57-79.

[19] Sanchez A, Trivech NS, Morrison DE. Preparation of the patient for awake intubation. In: Benumof JL, editor. Airway management: principles and practice. St. Louis: Mosby-Year Book; 1996. p. $159-82$.

[20] Barnett G, Hawks R, Resnick R. Cocaine pharmacokinetics in humans. J Ethnopharmacol 1981;3:353-66.

[21] Cone EJ. Pharmacokinetics and pharmacodynamics of cocaine. J Anal Toxicol 1995;19:459-78.

[22] Daniel WC, Mark PJ, Horton RP, et al. Electrophysiologic effects of intranasal cocaine. Am J Cardiol 1995;76:398-400.

[23] Greinwald JH, Holtel MR. Absorption of topical cocaine in rhinologic procedures. Laryngoscope 1996;106:1223-5.

[24] Hecker RB, Hays JV, Champ JD, et al. Myocardial ischemia and stunning induced by topical intranasal phenylephrine pledgets. Mil Med 1997;162:832-5.

[25] Jeffcoat A, Perex-Reyes M, Hill J, et al. Cocaine disposition in humans after intravenous injection, nasal insufflation (snorting), or smoking. Drug Metab Dispos 1989;17:153-9.

[26] Kalyanaraman M, Carpenter RL, McGlew MJ, et al. Cardiopulmonary compromise after use of topical and submucosal $\alpha$-agonist: possible added complication by the use of $\beta$-blocker therapy. Otolaryngol Head Neck Surg 1997;117:56-61.

[27] Kumar V, Schoenwald R, Barcellos W, et al. Aqueous vs. viscous phenylephrine: systemic absorption and cardiovascular effects. Arch Ophthalmol 1986;104:1189-91.

[28] Lange RA, Hillis LD. Cardiovascular complications of cocaine use. N Engl J Med 2001;345: $351-8$.

[29] Vongpatanasin W, Lange RA, Hillis LD. Comparison of cocaine-induced vasoconstriction of left and right coronary arterial systems. American Journal of Cardiology 1997;492-3.

[30] Agerton T, et al. Transmission of a highly drug-resistant strain (strainW) of Mycobacterium tuberculosis: community outbreak and nosocomial transmission via a contaminated bronchoscope. JAMA 1997;278:1073-7.

[31] Bronowicki JP, Venard V, Botte C. Patient-to-patient transmission of hepatitis C virus during colonoscopy. N Engl J Med 1997;337:237-40.

[32] Michele TM, Cronin WA, Graham NM. Transmission of Mycobacterium tuberculosis by a fiberoptic bronchoscope. JAMA 1997;278:1093-5.

[33] Hughes SC, et al. Recommendations for infection control for the practice of anesthesia. ASA 1998

[34] Martin MA, Reichelderfer M. APIC guidelines for infection prevention and control in flexible endoscopy. Am J Infect Control 1994;22:19-38.

[35] Rutala WA. APIC guideline for selection and use of disinfectants. Am J Infect Control 1996;24: $313-42$. 